\title{
Proposição de uma Escala de Consumo de Objetos Artesanais
}

\section{Proposal of a Handicraft Consumption Scale}

\author{
Rebeca da Rocha Grangeiro \\ Universidade Federal do Cariri - UFCA - Brasil \\ rebeca.grangeiro@ufca.edu.br \\ ORCID: 0000-0002-9292-2648 \\ Jailson Santana Carneiro \\ Universidade Federal Rural de Pernambuco - UFRPE - Brasil \\ jailson192@gmail.com \\ ORCID: 0000-0001-8182-7322
}

Lucas Emmanuel Nascimento Silva

Universidade Federal do Cariri - UFCA - Brasil lucas.emmanuel@aluno.ufca.edu.br ORCID: 0000-0001-7767-1724

Manoel Bastos Gomes Neto

Universidade Federal do Cariri - UFCA - Brasil neto26bastos@gmail.com ORCID: 0000-0003-4400-5877

Submetido em 22/09/2019; Aprovado em 20/01/2020.

\section{Resumo}

Objetivo do estudo: 0 presente estudo possui como objetivo desenvolver e validar uma escala de consumo de objetos artesanais. Metodologia/Abordagem: Utilizou-se de estudos da área de marketing e de entrevistas com seis consumidores de artesanato para ajudar na elaboração dos itens, que passaram pela validação de juízes. As amostras da primeira e da segunda coleta compreenderam, respectivamente, 209 e 442 respondentes. Foram realizadas análises fatoriais e de correlação e extração do Alpha. Adicionalmente, para a segunda coleta foi realizada Análise Fatorial Confirmatória, a partir da utilização do software AMOS. Principais resultados: Como resultado, obtivemos uma escala composta por quatro dimensões, cada uma com quatro itens. Duas dimensões pertencem ao momento pré-compra e dizem respeito às influências e características do bem artesanal que motivaram a compra; a terceira dimensão se encaixa no momento da compra propriamente dito e se refere ao serviço; a quarta remete ao momento posterior à compra e versa sobre a divulgação do bem adquirido. Contribuições práticas: $\mathrm{A}$ escala pode ser usada para mensuração do consumo de artesanatos de maneira geral e em contexto mais específico, desde que as devidas adaptações sejam feitas. Contribuições sociais: Em relação às implicações gerenciais, esse instrumento contribui, especialmente, para o conhecimento sobre o consumo de um produto cultural. Também se constitui como uma alternativa para os profissionais que querem saber mais sobre o consumo de suas peças, bem como gestores públicos que possam realizar pesquisas relacionadas ao turismo. Originalidade: A pesquisa se justifica por sua originalidade, uma vez que não foi encontrada na literatura medida com propósito semelhante.

Palavras-chave: desenvolvimento de escala; consumo de bens culturais; artesanato

\section{Abstract}

Study purpose: This study aims to develop and validate a scale of consumption of handcrafted objects. Methodology/ approach: Interviews were conducted with six handicraft consumers were conducted and the literature of the area was used to inspire the elaboration of the items, which were validated by experts. The samples from the first and second collections comprised respectively 209 and 442 respondents. Factorial and correlation analysis were performed and Alpha extraction. Additionally, for the second collection, Confirmatory Factor Analysis was performed using the AMOS software. Main 
findings: As results, we obtained a scale composed by four dimensions, each with four items. Two dimensions belong to the pre-purchase moment and concern the influences and characteristics of the artisanal good that motivated the purchase; the third dimension fits in with the purchase moment and refers to the service; the fourth refers to the moment after the purchase and deals with the disclosure of the acquired good. Practical implications: The scale can be used to measure handicraft consumption more generally and in a more specific context, provided that appropriate adjustments are made. Social implications: Regarding managerial contributions, this instrument contributes, especially, to the knowledge about the consumption of such a characteristic and specific cultural product. It also emerges as an alternative for professionals who want to know more about the consumption of their pieces, as well as public managers who can perform tourism-related researches. Originality: The research is justified by its originality, since it was not found in the literature measured with similar purpose.

Keywords: Scale development; consumption of cultural goods; handicraft

\section{Introdução}

Os bens culturais começaram a ganhar notoriedade a partir de uma nova concepção sobre as práticas e processos de consumo, decorrentes da transformação social, econômica e tecnológica (Mccrancken, 2003; Barbosa \& Campbell, 2006; Campbell, 2006). Segundo Silva, Araújo e Souza (2007), os bens culturais suprem lacunas materiais e culturais, possuem como função gerar informação e divertimento, mas também posicionar socialmente as pessoas. Assim, o consumo de bens culturais seria igualmente objeto de marcação e distinção social entre as pessoas, reproduzindo hábitos de consumo que manifestam o pertencimento a determinados grupos (Canclini, 1983).

Com o consumo de produtos artesanais não é diferente, a troca realizada é marcada por processos ainda pouco compreendidos e que carecem de maior investigação, especialmente sob a lente dos estudos de marketing, mais especificamente do comportamento do consumidor devido ao histórico dos produtos desta natureza.

Ao longo do tempo, observa-se a invasão de produtos industrializados que possuem baixo custo de produção, design considerado mais moderno e que acabam por substituir os objetos artesanais (Novaes, 2011; Keller, 2014). De fato, a expansão de produtos industrializados provoca a redução do consumo de objetos artesanais. No entanto, mesmo em período histórico denominado pós-industrial, observa-se a produção e o consumo destes. No caso de objetos artesanais altamente sofisticados e com altos preços de mercado, pode-se até mesmo falar em expansão da comercialização (Lima, 2009; Lages, Lagares, \& Braga, 2006).

Diante de todas essas transformações, identificam-se também mudanças no propósito do consumo ou no motivo que leva ao consumo de um objeto artesanal. Se em período anterior à expansão industrial, os objetos artesanais permeavam todo o dia a dia dos indivíduos, após esse período o consumo se dá por outros motivos, que não utilitários.

Assim, a finalidade do consumo também passa por transformações. Os objetos utilitários, lúdicos e religiosos ganham cunho acentuadamente decorativo, podendo em alguns momentos se aproximar de objetos artísticos e dividir com eles o espaço de museus. 0 artesanato é substituíd o por produtos industrializados nas demandas cotidianas e utilitárias da população local. Porém, continua sendo produzido, uma vez que passa a ser consumido por pessoas estrangeiras ao local de origem do objeto, para quem o propósito do consumo é menos utilitário e mais decorativo e/ou de expressão da subjetividade (Canclini, 1983). Dessa forma, o artesanato passa a ser mais consumido por turistas que pelos próprios habitantes de determinado local (Scrase, 2003; Bento \& Silva, 2016).

Segundo Canclini (1983), a justificativa para os turistas serem um dos grandes consumidores das peças artesanais relaciona-se ao conceito de souvenir, que são peças que remetem a lembranças de um determinado local visitado, com o objetivo de fazer o turista levar consigo recordações através de um objeto, aumentando assim a probabilidade de sua compra. Os viajantes, além de buscarem atestar a viagem a determinada localidade através do artesanato, também buscam demonstrar que seu gosto é amplo, ao ponto de abranger peças primitivas oriundas da produção das culturas populares (Canclini, 1983).

Nesse sentido, para uma melhor compreensão da compra de artesanato, algumas pesquisas têm se direcionado às estratégias utilizadas pelos artesãos (cf. Girón, Hernández, \& Castañeda, 2007; Mendoza- Ramírez \& Toledo-López, 2014) e outras se orientado mais para o consumo desses bens sob a 
ótica do consumidor, considerando características demográficas e construtos que influenciam a intenção de compra (cf. Silver \& Kundu, 2013; Pani \& Pradhan, 2016; Meitiana, Setiawan, Rohman \& Irawanto, 2019). 0 presente estudo se enquadra na segunda vertente, uma vez que analisará o consumo a partir de uma pesquisa com consumidores. Entretanto, nossos esforços consistem no desenvolvimento e validação de uma escala para consumo de objetos artesanais e não em teste de hipóteses.

Dito isso, e perante a ausência de pesquisas científicas que apresentem instrumento para mensuração de características pertinentes ao consumo de objetos artesanais, o presente estudo possui como objetivo desenvolver e validar uma escala de consumo de objetos artesanais. Tal pesquisa se justifica primeiramente por sua originalidade, uma vez que não encontramos na literatura medida com propósito semelhante, mesmo após buscas sistemáticas nas bases Scopus, Web of Science, Spell e Scholar Google. Em segundo lugar, é notório que apesar de ser uma atividade que gera ganhos econômicos e estar presente em mais de $70 \%$ dos municípios brasileiros, ela ainda sofre com a falta de conhecimento de gestão por parte dos artesãos (IBGE, 2015). Na perspectiva de estudos em comportamento do consumidor, a pesquisa contribui no direcionamento de esforços para a compreensão das motivações de compra dos consumidores de produtos artesanais, que são frequentemente negligenciadas, podendo ajudar na geração de insights para o entendimento do fenômeno do consumo desse segmento específico de produto. Em uma abordagem gerencial, este trabalho propõe entender os motivos que levam os consumidores à compra dos objetos artesanais, sendo assim, os artesãos podem identificar motivações de seu público alvo com o intuito de adaptar suas estratégias gerenciais de comercialização.

\section{Consumo}

O consumo surgiu como um fenômeno exclusivamente econômico (Cleps, 2004), entretanto, vários estudos revelaram a existência de um condicionamento cultural presente nas suas motivações (Reeves \& Vries, 2019; Montoro-Pons, 2018; Cleps, 2014; Katz-Gerro, 2002). Ademais, o consumo também é visto como uma dimensão do relacionamento social, que não se resume em comprar, desfrutar e descartar o produto, mas está condicionada a um encadeamento de informações que associa significados sócio-relacionais ao produto comercializado (Cruz, 2008).

Dessa forma, os bens que são consumidos possuem significados distintos para pessoas diferentes e entender as variantes de interpretação do consumidor sobre o produto é importante para os estudos sobre comportamento do consumidor (Cruz, 2008; Solomon, 2016). Assim, a escala busca compreender exatamente essas necessidades no momento pré-compra, momento da compra e no pós-compra que são relevantes no processo de decisão de compra de objetos artesanais.

\subsection{Comportamento pré-compra}

Os produtos artesanais possuem características que intrinsecamente tornam suas motivações de compra diferentes de outros produtos. As posses são uma forma conveniente de guardar as memórias e sentimentos conectados ao nosso passado, sendo assim, um souvenir pode ser usado como forma de tornar tangível uma experiência intangível (Belk, 1988; Pani \& Pradhan, 2016). Stewart (1993) apresenta como motivação para a compra de objetos feitos à mão, em detrimento a produtos industrialmente massificados, o fato de eles demoraram mais a serem produzidos e estarem fortemente vinculados ao self do criador do produto.

Rabêlo Neto et al. (2014) apontam três antecedentes que exercem maior influência sobre o consumo de produtos culturais, são eles: o capital cultural, a preferência e a influência de grupos de referência. Sobre a preferência, é possível identificar que, atualmente, o consumo passa da visão de "ter" para possuir características de construção da identidade do indivíduo. Então, os consumidores compram para descobrir quem são (Campbell, 2006). Por se tratar de produtos artesanais, os consumidores que buscam comprar esses objetos, fazem-no por motivos que excedem a funcionalidade dos bens.

Bragaglia (2010) apresenta a busca pelo prazer emocional como uma das motivações para o consumo. Esta pode estar ou não relacionada ao papel de comunicação social, sendo assim, o consumidor pode buscar o produto com o intuito de obter inserção em um grupo ou distinção social a partir do status atrelado ao objeto. Dessa forma, o status e as características do bem são variáveis importantes quando se investiga a intenção de compra de objetos artesanais (Silver \& Kundu, 2013).

Ademais, outro fator importante quando se analisa o comportamento do consumidor é a influência dos grupos de referências, a exemplo de amigos e familiares (Silver \& Kundu, 2013; Solomon, 2016). 
0 pertencimento a um grupo de referência é um fator motivacional importante para a compra. Ante a discussão feita, definiram-se os seguintes fatores que influenciam a compra de produtos artesanais nesse estágio, conforme exposto no Quadro 1.

IDENTIDADE: Procura identificar se os consumidores compram objetos artesanais com o objetivo de construir e apresentar suas identidades.

FINALIDADE: Procura identificar o objetivo do consumidor ao comprar o objeto artesanal.

INFLUÊNCIA DOS GRUPOS DE REFERÊNCIA: Pretende identificar se os grupos de referência - amigos, família... - possuem papel na motivação de compra de objetos artesanais.

CARACTERÍSTICAS DO BEM ARTESANAL: Procura identificar como as características tangíveis e intangíveis do objeto artesanal impactam na motivação para comprar o objeto.

ARTESÃO: Busca identificar o impacto do artesão, de suas habilidades, conhecimento, comprovada maestria e até mesmo visibilidade como influenciadores na motivação de compra do objeto artesanal.

Quadro 1. Dimensões do estágio pré-compra

Fonte: Elaborado pelos autores (2019).

\subsection{Comportamento no momento da compra}

A dimensão de comportamento no momento da compra tem o objetivo de identificar como alguns fatores presentes na ocasião de compras são capazes de influenciar as decisões do consumidor. 0 primeiro fator relevante no contexto da compra é o preço. A decisão para comprar um produto leva primeiramente em consideração o dispêndio que será necessário para obtê-lo, com a intenção de maximizar a satisfação (Pani \& Pradhan, 2016). 0 custo do produto não se baseia mais apenas pela sua aparência, mas sim pela presença do valor que há inserido nele, sobretudo quando o objeto está investido de valor social e simbólico, como no caso do artesanato (Sharda \& Bhat, 2018).

Fatores situacionais e do ambiente também influenciam a decisão de compra de produtos artesanais, a exemplo da qualidade do produto, das conveniências na hora das compras, bem como da forma como o produto está exposto (Meitiana et al., 2019). Nesse sentido, foram definidas as seguintes dimensões para o estágio momento da compra, conforme Quadro 2.

PREÇO: Procura identificar se o preço do bem artesanal é uma variável levada em consideração no momento da compra.

PRODUTO: Busca determinar como as características do bem artesanal são determinantes durante o momento da compra.

SERVIÇO: Procura determinar a relevância do serviço prestado no momento da aquisição na compra do bem artesanal.

Quadro 2. Dimensões do estágio momento da compra

Fonte: Elaborado pelos autores (2019).

\subsection{Comportamento pós-compra}

No momento do comportamento pós-compra, busca-se identificar o feedback fornecido pelos consumidores sobre os artesãos e os objetos artesanais, compreendendo se as necessidades e expectativas dos consumidores foram supridas pela qualidade do bem e atendimento prestado.

0 consumo é um processo em que o indivíduo busca a satisfação de suas necessidades respeitando uma hierarquia de satisfação (Chagas Neto, 2006). Satisfação é definida como o resultado da avaliação da discrepância entre as expectativas e experiências com o consumo (Marchetti \& Prado, 2001). Contudo, Giese e Cote (2000) destacam que nem sempre os consumidores conseguem recordar suas expectativas e relacionam o seu grau de contentamento com o produto em três aspectos: i) resposta cognitiva e emocional, semelhante a uma somatória de respostas afetivas variando em relação a sua intensidade; ii) satisfação ao longo do tempo - desde o momento de escolha, durante e após o uso; e, iii) aspectos que influenciam a experiência de compra.

Artoni e Daré (2008) apontam que a satisfação com a experiência no ambiente de consumo é o estímulo para a geração da propaganda boca a boca. Essa divulgação, ou recomendação espontânea, é a troca de informações e disseminação de determinada marca, serviço ou produto através de meios interpessoais espontâneos (Sirdeshmukh, Singh \& Sabol, 2002). Assim, para o estágio pós-compra, foram consideradas como dimensões a Satisfação e a Divulgação, conforme se observa no Quadro 3. 
DIVULGAÇÃO: Busca determinar como os consumidores anunciam sua aquisição do objeto artesanal.

Quadro 3. Dimensões do estágio pós-compra

Fonte: Elaborado pelos autores (2019).

\section{Desenvolvimento da escala}

A operacionalização de desenvolvimento e validação da escala aqui pretendida seguiu o modelo proposto por Costa (2011). Nesse sentido, o autor supracitado elenca dez passos para a produção de uma escala consistente, confiável e válida para aplicação.

\section{Passo 1 - Especificação do domínio do construto}

Este primeiro momento consiste na definição clara e precisa do que se pretende mensurar. A escala proposta no presente trabalho tem como escopo examinar as motivações para o consumo de objetos artesanais, o comportamento do consumidor no ato da compra e em momento posterior. Esses três momentos (pré-compra, compra e pós-compra) foram o ponto de partida para a criação das dimensões que compõem a escala. Guiados por essa estrutura fundamentada em três momentos, a leitura de referenciais teóricos sobre artesanato, marketing e consumo de bens artísticos e/ou culturais auxiliou na criação de dez dimensões cujas definições foram expostas na seção do Referencial Teórico.

\section{Passo 2 - Atividades de geração de itens e validação de face e conteúdo}

Os itens foram gerados a partir de três estratégias. Após identificação de cada uma delas, apresentamos o exemplo de um ou dois itens da escala que foi construído seguindo essa estratégia. A primeira diz respeito à adaptação de itens de escalas existentes, que avaliam consumo de bens artísticos e/ou culturais;

\footnotetext{
Item oriundo da escala criada por Rabêlo Neto et al. (2014) - Busco experiência e conhecimento com amigos, vizinhos, parentes ou colegas de trabalho que têm informações confiáveis sobre cultura.

Item adaptado para escala de consumo de objetos artesanais - Quando um amigo me apresenta um produto artesanal, eu me sinto tentado a comprá-lo.
}

A segunda estratégia fez uso do referencial teórico acessado. Então, a partir de sua leitura e compreensão, o item foi elaborado;

Souza (1993, p. 34) afirma: " O artesanato é um produto que expressa a cultura de uma região ou país".

Então, elaboramos o item - Eu compro artesanato porque expressa a cultura da região/país de origem do bem.

A última estratégia utilizada foi a condução de entrevistas semi-estruturadas com consumidores de artesanato. As entrevistas foram realizadas, presencialmente, com o consentimento dos seis participantes que foram acessados em lugares públicos, principalmente, próximo a pontos tradicionais de comercialização de artesanato. As entrevistas foram gravadas e transcritas. Uma vez que esta foi uma etapa concretizada quando 50 itens já estavam elaborados, os depoimentos dos entrevistados já estavam vislumbrados na escala construída, exceto por dois comentários que influenciaram a elaboração de mais dois itens, detalhados a seguir.

\footnotetext{
Trecho da Entrevista 01 -"Eu... muito percebo a qualidade quando vejo eles fazendo, são bons e bonitos e quando eu chego em algum lugar o pessoal já olha..."

Item criado - Eu observo a qualidade do objeto artesanal no momento da compra.

Trecho da Entrevista 05 - "Essa coisa da matéria-prima é um atrativo pra mim né, então, objetos produzidos com palha, com madeira, com couro."

Item criado - A matéria-prima com a qual é feito o objeto me influencia no momento da compra.
}

Definidos os 52 itens da escala, realizou-se validação de face e conteúdo da escala, entre os meses de março e abril de 2019, a partir da avaliação de dez juízes quanto à clareza dos enunciados e adequação dos itens às dimensões propostas. Os juízes especialistas no tema eram todos mestres ou doutores da área de marketing. Após essa avaliação, um item foi excluído e, seguindo sugestão dos avaliadores, nove itens foram levemente alterados para que ficassem mais compreensíveis para os respondentes. 


\section{Passo 3 - Decisões sobre as respostas}

Neste passo foi analisada a forma como a escala foi verificada. Para o contexto aqui pretendido, foi escolhida pelos pesquisadores a escala Likert de sete pontos, a qual variava de 1 (Discordo totalmente) a 7 (Concordo totalmente) nas afirmações.

\section{Passo 4 - Construção do Instrumento de Pesquisa}

O questionário foi estruturado de modo que os itens de cada construto ficassem misturados para evitar viés de respostas dos inquiridos. Para garantir que apenas consumidores de objetos artesanais respondessem à escala, logo no início do questionário foi perguntado se o respondente consumiu ou consome objetos artesanais. Caso a resposta fosse negativa, o questionário era concluído, caso fosse positiva, outras questões eram apresentadas. Ainda, os dados sociodemográficos foram inseridos aproximadamente na metade do questionário, dividindo a escala em duas partes a fim de evitar efeito halo. Terminada essa etapa, foi feita a aplicação do pré-teste com 12 respondentes (estudantes universitários) com o objetivo de verificar eventuais equívocos e não compreensão dos itens. Dessa forma, não houve modificações necessárias e se procedeu a coleta de dados.

\section{Passo 5 - Primeira atividade de amostragem}

Este passo tem como intuito analisar a escala de maneira exploratória. Assim, procedemos à coleta dos dados, que foi feita por conveniência e acessibilidade dos pesquisadores de modo presencial e online. 0 questionário online foi desenvolvido na plataforma Survey Monkey e o link foi compartilhado em redes sociais, Facebook, Instagram e Whatsapp dos autores e de outros pesquisadores não envolvidos diretamente neste estudo. Para esse primeiro momento, a coleta de dados se deu entre abril e maio de 2019. Depois de coletados, realizou-se a tabulação dos dados aplicados de maneira presencial junto aos dados online no SPSS (Statistical Package for The Social Sciences) versão 22, software usado para análises estatísticas. Todos os procedimentos foram operacionalizados no SPSS e baseados na literatura especializada (Field, 2009; Hair et al., 2009; Costa, 2011).

\section{Passo 6 - Primeiros procedimentos de limpeza da escala}

Os primeiros procedimentos de limpeza consistiram na verificação de missing values e outliers. Obteve-se 49 questionários através de aplicação presencial e 214 por meio de coleta online. Para a coleta presencial, os questionários foram impressos e entregues a pessoas que aceitavam respondê-lo. Essas pessoas foram acessadas em feiras de artesanato de quatro cidades do estado do Ceará, e instituições de ensino superior. Nessa etapa, 36 questionários, oriundos das duas formas de coleta de dados, foram excluídos por não estarem completamente respondidos, restando 209 questionários válidos. A amostra foi composta por $55 \%$ de mulheres e $45 \%$ de homens, a idade dos respondentes variou entre 16 e 54 anos, com média 23,12 anos e desvio padrão de 6,53. A maior parte da amostra possui ensino médio $(53,1 \%)$ e superior $(23,9 \%)$ completo. Quanto à renda, $82,8 \%$ dos respondentes afirmaram possuir renda de até dois salários mínimos por mês. Os respondentes residem em 19 municípios dos estados do Ceará e Pernambuco. Após essas análises preliminares, realizamos as análises exploratórias por meio das quais extraímos as medidas de correlação, consistência interna e análise fatorial.

\section{Estágio Pré-compra}

\section{Dimensão de Identidade}

Por meio da análise de correlação verificamos que todas as medidas, apesar de significativas, ficaram em níveis baixos. A exceção foi a correlação dos itens PRE_IDEN3 e PRE_IDEN4 que obteve um valor moderado de 0,417 . Os dados estão dispostos na Tabela 1 .

Tabela 1: Matriz de correlação do estágio Pré-compra, dimensão Identidade

\begin{tabular}{|c|c|c|c|}
\hline & PRE_IDEN2 & PRE_IDEN3 & PRE_IDEN4 \\
\hline PRE_IDEN1 & ,279** & ,209** & $173^{*}$ \\
\hline PRE_IDEN2 & & ,270** & ,356** \\
\hline PRE_IDEN3 & & &, $417^{* *}$ \\
\hline
\end{tabular}

Para verificar a confiabilidade da escala, extraímos o alpha de Cronbach. Com os quatro itens, obteve-se o valor de 0,616. Com a exclusão do item PRE_IDEN1, ele chegava a 0,617, representando valores pouco acima do mínimo aceitável (Costa, 2011). Decidimos não excluir o item de imediato, mas verificar 
como ele se comportaria na análise fatorial. Em um primeiro momento, extraímos as medidas com os quatro itens, entretanto a variância explicada ficou em 46,62\%. Ao verificar as comunalidades e escores fatoriais, visualizamos que os menores valores são referentes ao item PRE_IDEN1 (sendo 0,302 para comunalidade e 0,550 para escore fatorial). Nesse momento, procedemos à exclusão deste para melhorar as medidas, especialmente a variância explicada. 0 teste de adequação da amostra $(K M 0=0,604)$ e de esfericidade de Bartlett $\left(\chi^{2}=36,764 \mathrm{gl}=3\right.$, a $\left.\mathrm{p}<0,000\right)$ sinalizaram que os itens estavam adequados para a análise fatorial, ficando a variância em 50,24\%, conforme dados da Tabela 2.

Tabela 2: Medidas da análise fatorial do estágio Pré-compra, dimensão Identidade

\begin{tabular}{clcc}
\hline \multirow{2}{*}{ CóD } & & \multicolumn{1}{c}{ Item } & \multicolumn{2}{c}{ Variância: 50,24\% } \\
\cline { 3 - 4 } & & Comu & Esc \\
\hline PRE_IDEN1 & Eu compro artesanato porque revela quem eu sou & $*$ & $*$ \\
PRE_IDEN2 & Eu compro artesanato porque é um produto único &, 481 &, 694 \\
PRE_IDEN3 & Eu compro artesanato porque quero me diferenciar &, 558 &, 747 \\
PRE_IDEN4 & Eu compro artesanato pois não gosto de bens consumidos por muitas pessoas &, 469 &, 684 \\
\hline
\end{tabular}

Nota. ${ }^{*}$ Item deletado. Fonte: Dados da pesquisa (2019).

\section{Dimensão de Finalidade}

Na dimensão Finalidade, boa parte das correlações foram nulas, baixas ou negativas, variando de $-0,066$ a 0,132 . Apenas quatro foram significativas, sendo uma moderada positiva $(0,449)$, duas baixas positivas $(0,358$ e 0,181$)$ e uma baixa negativa $(-0,151)$. Tais resultados sinalizam possíveis problemas com os itens, conforme os dados dispostos na Tabela 3.

Tabela 3: Matriz de correlação do estágio Pré-compra, dimensão finalidade

\begin{tabular}{|c|c|c|c|c|c|}
\hline & PRE_FIN2 & PRE_FIN3 & PRE_FIN4 & PRE_FIN5 & PRE_FIN6 \\
\hline PRE_FIN1 & ,449** &,- 066 & ,358** & ,181** &,- 029 \\
\hline PRE_FIN2 & & -038 & 109, & 132 & 080 \\
\hline PRE_FIN3 & & &,$- 151^{*}$ & 051 & 130 \\
\hline PRE_FIN4 & & & & 101 &,- 031 \\
\hline PRE_FIN5 & & & & & ,081 \\
\hline
\end{tabular}

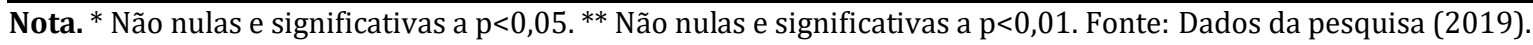

$\mathrm{Na}$ análise fatorial, dois fatores apresentaram autovalores maiores que 1, denotando que os itens de fato não convergem bem entre si. A análise consistência interna por meio do alpha de Cronbach também reforçou os problemas com a dimensão Finalidade, ficando em 0,344. Então, procedemos para exclusões de itens com o intuito de melhorar as medidas da escala, sendo que inicialmente foi verificado o enunciado de cada variável. Posteriormente, foram excluídas as variáveis PRE_FIN3, PRE_FIN5 e PRE_FIN6, considerando os resultados das correlações, comunalidades e escores fatoriais. 0 teste de adequação da amostra (KMO $=0,517)$ e de esfericidade de Bartlett $\left(\chi^{2}=75,484 \mathrm{gl}=3\right.$, a $\left.\mathrm{p}<0,000\right)$ sinalizaram que os itens estavam adequados para a análise fatorial. Os resultados estão expostos na Tabela 4.

Tabela 4: Medidas da análise fatorial do estágio Pré-compra, dimensão Finalidade

\begin{tabular}{|c|c|c|c|}
\hline \multirow[t]{2}{*}{ CóD } & \multirow[t]{2}{*}{ Item } & \multicolumn{2}{|c|}{$\begin{array}{l}\text { Variância: } 54,35 \% \\
\text { Alpha: } 0,555\end{array}$} \\
\hline & & Comu & Esc \\
\hline PRE_FIN1 & Eu compro artesanato para recordar o local visitado & 0,739 & 0,860 \\
\hline PRE_FIN2 & $\begin{array}{l}\text { Eu compro um objeto artesanal como uma maneira de comprovar uma viagem re- } \\
\text { alizada }\end{array}$ & 0,516 & 0,719 \\
\hline PRE_FIN3 & Eu compro artesanato para utilizar no dia a dia & $*$ & $*$ \\
\hline PRE_FIN4 & Eu compro artesanato para fins decorativos & 0,375 & 0,613 \\
\hline PRE_FIN5 & Eu compro artesanato para ajudar o artesão no seu ofício & * & * \\
\hline PRE_FIN6 & Eu compro artesanato para fins recreativos & $*$ & $*$ \\
\hline
\end{tabular}


Ainda de acordo com os dados da Tabela 4, a variância nessa nova extração ficou em 54,35\% comparada com a primeira, que havia ficado em $49,14 \%$. Já a consistência interna ficou em 0,555 , valor considerado inferior ao mínimo aceitável de 0,600.

\section{Dimensão de Influência dos grupos de referência}

Considerando as análises para a dimensão Influência, notamos que todas as medidas ficaram em níveis adequados. Todas as correlações foram significativas e positivas, sendo que a de menor valor de 0,368 . As demais ficaram em níveis moderados, conforme exposto na Tabela 5 .

Tabela 5: Matriz de correlação do estágio Pré-compra, dimensão Influência dos grupos de referência

\begin{tabular}{lccc}
\hline & PRE_INFLU2 & PRE_INFLU3 & PRE_INFLU4 \\
\hline PRE_INFLU1 &, $593^{* *}$ &, $368^{* *}$ &, $453^{* *}$ \\
PRE_INFLU2 & &, $470^{* *}$ &, $561^{* *}$ \\
PRE_INFLU3 & & &, $409^{* *}$ \\
\hline
\end{tabular}

Nota. $^{* *}$ Não nulas e significativas a p<0,01. Fonte: Dados da pesquisa (2019).

$\mathrm{Na}$ análise consistência interna, não haveria melhoras do valor de alpha de Cronbach, caso alguma variável fosse deletada, ficando a medida em 0,782. 0 teste de adequação da amostra também sinalizou que os itens eram adequados para a análise fatorial com teste $\mathrm{KMO}=0,768$ e de esfericidade de Bartlett $\left(\chi^{2}=235,177 \mathrm{gl}=6\right.$, a $\mathrm{p}<0,000$. A variância extraída foi de $60,91 \%$. Na Tabela 6 , apresentamos os valores referentes à análise fatorial exploratória.

Tabela 6: Medidas da análise fatorial do estágio Pré-compra, Influência dos grupos de referência

\begin{tabular}{|c|c|c|c|}
\hline \multirow[t]{2}{*}{ CóD } & \multirow[t]{2}{*}{ Item } & \multicolumn{2}{|c|}{$\begin{array}{l}\text { Variância: } 60,91 \% \\
\text { Alpha: } 0,782 \\
\end{array}$} \\
\hline & & Comu & Esc \\
\hline PRE_INFLU1 & $\begin{array}{l}\text { Quando vejo um amigo com um produto artesanal eu me sinto motivado a comprar } \\
\text { o produto }\end{array}$ & 0,606 & 0,779 \\
\hline PRE_INFLU2 & $\begin{array}{l}\text { Quando um amigo me apresenta um produto artesanal, eu me sinto tentado a com- } \\
\text { prá-lo }\end{array}$ & 0,731 & 0,855 \\
\hline PRE_INFLU3 & $\begin{array}{l}\text { Quando um membro da minha família indica um produto artesanal, eu me sinto ten- } \\
\text { tado a comprá-lo }\end{array}$ & 0,492 & 0,701 \\
\hline PRE_INFLU4 & $\begin{array}{l}\text { Quando vejo uma postagem sobre artesanato nas redes sociais, eu sinto desejo em } \\
\text { comprá-lo }\end{array}$ & 0,608 & 0,779 \\
\hline
\end{tabular}

Fonte: Dados da pesquisa (2019).

\section{Dimensão de Características do bem artesanal}

Seguindo com os mesmos procedimentos adotados anteriormente, percebe-se que a dimensão Características do bem artesanal apresenta muitas correlações não significativas. Entre as significativas, as medidas se situam em níveis baixos $(0,162$ a 0,357$)$ ou moderados $(0,415$ a 504$)$. Isso é um primeiro indício de que alguns itens precisam ser excluídos para uma melhor adequação. Os dados completos das correlações estão dispostos na Tabela 7.

Tabela 7: Matriz de correlação do estágio Pré-compra, dimensão Características do bem material

\begin{tabular}{|c|c|c|c|c|c|c|c|c|}
\hline & $\begin{array}{c}\text { PRE_C } \\
\text { AR2 }\end{array}$ & $\begin{array}{l}\text { PRE } \\
\text { CAR3 }\end{array}$ & $\begin{array}{c}\text { PRE }_{-} \\
\text {CAR4 }\end{array}$ & $\begin{array}{l}\text { PRE }_{-} \\
\text {CAR5 }\end{array}$ & $\begin{array}{l}\text { PRE }_{-} \\
\text {CAR6 }\end{array}$ & $\begin{array}{l}\text { PRE } \\
\text { CAR7 }\end{array}$ & $\begin{array}{l}\text { PRE } \\
\text { CAR8 } \\
\end{array}$ & $\begin{array}{l}\text { PRE } \\
\text { CAR9 } \\
\end{array}$ \\
\hline PRE_CAR1 &,- 080 & ,268** & ,067 & 103 & ,073 & ,193** & ,012 &,- 041 \\
\hline PRE_CAR2 & &, 044 & 083 & 110 &, $263^{* *}$ & ,239** & ,169* & ,095 \\
\hline PRE_CAR3 & & &, $347^{* *}$ & $173^{*}$ &, $162^{*}$ &, $282^{* *}$ &,- 014 & 106 \\
\hline PRE_CAR4 & & & &, $504^{* *}$ &, $438^{* *}$ & ,281** & 070 &, $357^{* *}$ \\
\hline PRE_CAR5 & & & & &, $415^{* *}$ &, $342^{* *}$ & ,126 & $284^{* *}$ \\
\hline PRE_CAR6 & & & & & & ,179** & ,094 &, $243^{* *}$ \\
\hline PRE_CAR7 & & & & & & & ,014 & $185^{* *}$ \\
\hline PRE_CAR8 & & & & & & & &, $205^{* *}$ \\
\hline
\end{tabular}

Nota. ${ }^{*}$ Não nulas e significativas a p<0,05. ** Não nulas e significativas a p<0,01. Fonte: Dados da pesquisa (2019).

Na análise de confiabilidade, o valor do alpha de Cronbach ficou em 0,663 com os nove itens, apesar das fracas correlações, a medida pode ter ficado acima do nível mínimo exigido $(0,600)$ por conta do alto número de itens (Costa, 2011). A análise fatorial mostrou que três fatores ficaram com autovalores maiores que 1 , sinalizando que os itens não estão mensurando adequadamente a dimensão pretendida. 
Assim, considerando o enunciado dos itens, o escore fatorial, a comunalidade e o indicativo de melhoria do alpha com exclusão de itens, excluímos as variáveis PRE_CAR1, PRE_CAR2 e PRE_CAR8. 0 novo alpha ficou em 0,704 com seis itens, conforme dados expostos na Tabela 8.

Tabela 8: Medidas da análise fatorial do estágio Pré-compra, dimensão Características do bem material

\begin{tabular}{|c|c|c|c|}
\hline \multirow[t]{2}{*}{ CÓD } & \multirow[t]{2}{*}{ Item } & \multicolumn{2}{|c|}{$\begin{array}{c}\text { Variância: } 41,42 \% \\
\text { Alpha: } 0,704\end{array}$} \\
\hline & & Comu & Esc \\
\hline PRE_CAR1 & Eu prefiro comprar objetos artesanais feitos no estado onde moro & * & * \\
\hline PRE_CAR2 & Compro artesanato quando visito outras cidades & $*$ & $*$ \\
\hline PRE_CAR3 & Eu compro artesanato por não ser uma produção industrial massificada & ,241 & ,491 \\
\hline PRE_CAR4 & $\begin{array}{l}\text { Eu compro artesanato por envolver uma habilidade técnica repassada por ge- } \\
\text { rações }\end{array}$ & ,640 & 800 \\
\hline PRE_CAR5 & Eu compro artesanato por envolver habilidade manual & 557 & ,747 \\
\hline PRE_CAR6 & $\begin{array}{l}\text { Eu compro artesanato porque expressa a cultura da geração/país de origem } \\
\text { do bem }\end{array}$ & ,430 & ,656 \\
\hline PRE_CAR7 & Eu compro artesanato porque é sustentável & 318 &, 564 \\
\hline PRE_CAR8 & Eu compro artesanato porque o produto é bonito & $*$ & $*$ \\
\hline PRE_CAR9 & $\begin{array}{l}\text { Eu compro artesanato pois representa a combinação entre a tradição e a mo- } \\
\text { dernidade }\end{array}$ & ,300 & ,547, \\
\hline
\end{tabular}

Nota. *Item deletado. Fonte: Dados da pesquisa (2019).

0 teste de adequação da amostra ficou com a seguinte medida $\mathrm{KMO}=0,752$ e o de esfericidade de Bartlett $\chi^{2}=215,881 \mathrm{gl}=15$, a $\mathrm{p}<0,000$. Entretanto, a variância ficou em $41,42 \%$ considerando as exclusões, conforme se observa na Tabela 8. Como essa foi a única medida fora dos padrões, preferimos manter a dimensão para a próxima coleta. Alguns itens também apresentaram escores abaixo de 0,500, contudo optamos por deixá-los para verificar os seus comportamentos na segunda coleta de dados.

\section{Dimensão do Artesão}

Quando analisamos as correlações para a dimensão referente ao Artesão, notamos que apesar de positivas, algumas não foram significantes ficando em valores muito baixos $(0,034 ; 0,080 ; 0,095$ e $0,117)$. Outras foram significantes em nível baixo e apenas duas alcançaram medidas moderadas $(0,402$ e 0,414 ), conforme dados da Tabela 9.

Tabela 9: Matriz de correlação do estágio Pré-compra, dimensão Artesão

\begin{tabular}{lcccc}
\hline & PRE_ART2 & PRE_ART3 & PRE_ART4 & PRE_ART5 \\
\hline PRE_ART1 &, $230^{* *}$ &, 117 &, 080 &, $402^{* *}$ \\
PRE_ART2 & &, $414^{* *}$ &, $311^{* *}$ &, $218^{* *}$ \\
PRE_ART3 & &, $336^{* *}$ &, 095 \\
PRE_ART4 & & & &, 034 \\
\hline
\end{tabular}

Nota. $^{* *}$ Não nulas e significativas a $\mathrm{p}<0,01$. Fonte: Dados da pesquisa (2019).

Os resultados das baixas correlações se refletiram na consistência interna da dimensão, que apresentou um valor inicial de 0,593. Considerando os parâmetros já mencionados para exclusão de itens, decidimos deletar as variáveis PRE_ART1 e PRE_ART5. 0 teste de adequação da amostra $(K M 0=0,637)$ e de esfericidade de Bartlett $\left(\chi^{2}=71,991 \mathrm{gl}=3\right.$, a $\left.\mathrm{p}<0,000\right)$ sinalizou que os itens estavam adequados para a análise fatorial. 0 novo valor do alpha ficou em 0,621 e a variância em $56,99 \%$, conforme dados da Tabela 10.

Tabela 10: Medidas da análise fatorial do estágio Pré-compra, dimensão Artesão

\begin{tabular}{|c|c|c|c|}
\hline \multirow[t]{2}{*}{ CóD } & \multirow[t]{2}{*}{ Item } & \multicolumn{2}{|c|}{$\begin{array}{l}\text { Variância: } 56,99 \% \\
\text { Alpha: } 0,621\end{array}$} \\
\hline & & Comu & Esc \\
\hline PRE_ART1 & Conhecer o trabalho do artesão me ajudou na decisão de compra & * & * \\
\hline PRE_ART2 & A fama do artesão é importante para que eu realize a compra & 0,591 & 0,769 \\
\hline PRE_ART3 & Prefiro comprar produtos de artesão renomado & 0,616 & 0,785 \\
\hline PRE_ART4 & $\begin{array}{l}\text { Eu não gosto de comprar objetos produzidos por um artesão que eu não } \\
\text { conheço }\end{array}$ & 0,503 & 0,709 \\
\hline PRE_ART5 & $\begin{array}{l}\text { O conhecimento que o artesão tem do produto é importante para que eu } \\
\text { realize a compra }\end{array}$ & $*$ & $*$ \\
\hline
\end{tabular}




\section{Estágio Momento da compra \\ Dimensão Preço}

A dimensão Preço foi mensurada a partir de três itens. As medidas de correlação mostram que apenas uma não foi significativa 0,119 . As demais, apesar de serem não nulas, ficaram em nível baixo $(0,377)$ e moderado $(0,452)$. Os resultados estão expostos da Tabela 11.

Tabela 11: Matriz de correlação do estágio Momento da compra, dimensão Preço

\begin{tabular}{ccc}
\hline & COM_PRE2 & COM_PRE3 \\
\hline COM_PRE1 & $0,452^{* *}$ & 0,119 \\
COM_PRE2 & & $0,377^{* *}$ \\
\hline Nota. ** Não nulas e significativas a p<0,01. Fonte: Dados da pesquisa (2019).
\end{tabular}

Na mensuração da consistência interna, a medida do alpha ficou em 0,555, inicialmente, com indicativo de melhoria se o item 3 fosse excluído. A variância extraída foi de 55,92\% e o menor escore foi do item COM_PRE3 $(0,630)$. Nesse sentido, optamos por deletar o referido item no intuito de obter melhores medidas. A amostra foi considerada adequada para a análise fatorial, considerando os resultados dos testes $\mathrm{KMO}=0,500$ e de esfericidade de Bartlett $\chi^{2}=47,264 \mathrm{gl}=1$, a $\mathrm{p}<0,000$. A variância com os dois itens ficou em 72,61\% e o alpha de Cronbach em 0,622. Os dados estão expostos na Tabela 12.

Tabela 12: Medidas da análise fatorial do estágio Momento da compra, dimensão Preço

\begin{tabular}{clcc}
\hline \multirow{2}{*}{ CÓD } & \multirow{2}{*}{ Item } & \multicolumn{2}{c}{ Variância: 72,61\% } \\
Alpha: 0,622 \\
\cline { 3 - 4 } COM_PRE1 & Eu prefiro comprar artesanato que possua preço justo & Comu & Esc \\
COM_PRE2 & O preço é importante para minha decisão de compra & 0,726 & 0,852 \\
COM_PRE3 & Ao buscar um produto artesanal, o preço é a primeira coisa que considero & 0,726 & 0,852 \\
\hline
\end{tabular}

Nota. ${ }^{*}$ Item deletado. Fonte: Dados da pesquisa (2019).

\section{Dimensão Produto}

A dimensão Produto era composta a princípio por quatro itens. As correlações foram todas significativas, porém, em sua maioria, em níveis baixos $(<0,300)$. Isso sinaliza eventuais problemas na mensuração da referida dimensão. Os dados das medidas de correlação estão expostos na Tabela 13, a seguir. Tabela 13: Matriz de correlação do estágio Momento da compra, dimensão Produto

\begin{tabular}{cccc}
\hline & COM_PRO2 & COM_PRO3 & COM_PRO4 \\
\hline COM_PRO1 &, $273^{* *}$ &, $309^{* *}$ &, $224^{* *}$ \\
COM_PRO2 &, $230^{* *}$ &, $242^{* *}$ \\
COM_PRO3 & &, $183^{* *}$ \\
\hline Nota. $^{* *}$ Não nulas e significativas a p<0,01. Fonte: Dados da pesquisa (2019).
\end{tabular}

Na verificação de confiabilidade da escala, o valor do alpha de Cronbach ficou em 0,545. A variância inicial em 43,34\%. Considerando os valores da correlação, da comunalidade $(0,359)$ e do escore fatorial $(0,599)$, deletamos a variável COM_PRO4. Com isso, a variância explicada subiu para 51,42\%. 0 teste de adequação da amostra (KMO=0,611) e de esfericidade de Bartlett $\left(\chi^{2}=41,971 \mathrm{gl}=3\right.$, a $\left.\mathrm{p}<0,000\right)$ sinalizaram adequação para a análise fatorial. Já o alpha sofreu uma leve redução e ficou em 0,519 após a exclusão da variável COM_PRO4, conforme dados da Tabela 14.

Tabela 14: Medidas da análise fatorial do estágio Momento da compra, dimensão Produto

\begin{tabular}{clcc}
\hline \multirow{2}{*}{ CÓD } & \multirow{2}{*}{ Item } & \multicolumn{2}{c}{ Variância: 51,42\% } \\
\cline { 3 - 4 } & & Comu & Esc \\
\hline COM_PR01 & Eu observo a qualidade do objeto artesanal no momento da compra & 0,564 & 0,751 \\
COM_PRO2 & Eu observo se existe inovação nos produtos do artesão & 0,465 & 0,682 \\
COM_PR03 & A matéria prima do objeto me influencia no momento da compra & 0,514 & 0,717 \\
COM_PRO4 & Eu não compro objetos artesanais de baixa qualidade & $*$ & $*$ \\
\hline
\end{tabular}

Nota. *Item deletado. Fonte: Dados da pesquisa (2019).

\section{Dimensão Serviço}

Considerando a dimensão Serviço, mensurada por meio de seis itens, percebe-se por meio dos 
resultados da Tabela 15, que as variáveis COM_SER4 e COM_SER6 apresentaram correlações nulas. Nesse sentido, temos o primeiro indicativo de que elas não convergem bem com os demais itens. As demais correlações, ainda que apresentando resultados baixos ou moderados, foram todas significativas.

Tabela 15: Matriz de correlação do estágio Momento da compra, dimensão Serviço

\begin{tabular}{lccccc}
\hline & COM_SER2 & COM_SER3 & COM_SER4 & COM_SER5 & COM_SER6 \\
\hline COM_SER1 &, $230^{* *}$ &, $222^{* *}$ &,- 033 &, $311^{* *}$ &, 037 \\
COM_SER2 & &, $354^{* *}$ &, 017 &, $279^{* *}$ &, 015 \\
COM_SER3 & &, 063 &, $190^{* *}$ &, 126 \\
COM_SER4 & & &, $161^{*}$ &, 038 \\
COM_SER5 & & & &, 134 \\
\hline
\end{tabular}

Nota. * Não nulas e significativas a p<0,05. ** Não nulas e significativas a p<0,01. Fonte: Dados da pesquisa (2019).

$\mathrm{Na}$ análise fatorial, observou-se que dois fatores apresentaram valores maiores que um. Ao analisar os escores fatoriais, os itens que apresentaram problemas na tabela de correlação foram os que obtiveram menores valores (COM_SER4=-0,077e COM_SER6=0,086). A consistência interna, inicialmente, apresentou valor de 0,450, bem inferior ao mínimo aceitável de 0,600 (Costa, 2011). Após a exclusão dessas duas variáveis, os testes de adequação da amostra $(\mathrm{KMO}=0,660)$ e de esfericidade de Bartlett $\left(\chi^{2}=76,220 \mathrm{gl}=6\right.$, a $\left.\mathrm{p}<0,000\right)$ sinalizaram que os itens estavam adequados para a análise fatorial. A variância extraída foi de $44,87 \%$ e o alpha de Cronbach ficou em 0,593, ambas ficaram abaixo do mínimo recomendável, 50\% e 0,600, respectivamente (Hair et al., 2005, Costa, 2011). Os dados completos podem ser visualizados na Tabela 16.

Tabela 16: Medidas da análise fatorial do estágio Momento da compra, dimensão Serviço

\begin{tabular}{llcc}
\hline \multirow{2}{*}{ CÓD } & \multirow{2}{*}{ Item } & \multicolumn{2}{c}{$\begin{array}{c}\text { Variância: } 44,87 \% \\
\text { Alpha: } 0,593\end{array}$} \\
\cline { 3 - 4 } & & Comu & Esc \\
\hline COM_SER1 & A forma como o objeto é exposto me incentiva a comprá-lo & 0,421 & 0,649 \\
COM_SER2 & Eu observo o atendimento prestado no momento da compra do artesanato & 0,507 & 0,712 \\
COM_SER3 & Costumo levar mais produtos artesanais quando sou bem atendido & 0,431 & 0,656 \\
COM_SER4 & Ao comprar objetos artesanais, observo se existe opção de troca & $*$ & $*$ \\
COM_SER5 & Eu observo o conforto do ambiente de compra para a aquisição do objeto artesanal & 0,436 & 0,660 \\
COM_SER6 & Já deixei de comprar uma peça artesanal porque o artesão só recebia em dinheiro & $*$ & $*$ \\
\hline
\end{tabular}

Nota. ${ }^{*}$ Item deletado. Fonte: Dados da pesquisa (2019).

\section{Estágio Pós-compra}

\section{Dimensão Satisfação}

No estágio Pós-compra, a dimensão Satisfação foi mensurada inicialmente com quatro itens. As correlações ficaram em níveis baixos e variaram de 0,083 a 0,358 , sendo que a mais baixa foi nula. As demais foram significativas. De maneira geral, a variável POS_SAT4 foi a que apresentou as menores medidas, conforme dados da Tabela 17.

Tabela 17: Matriz de correlação do estágio Pós-compra, dimensão Satisfação

\begin{tabular}{cccc}
\hline & POS_SAT2 & POS_SAT3 & POS_SAT4 \\
\hline POS_SAT1 &, $358^{* *}$ &, $253^{* *}$ &, 083 \\
POS_SAT2 & &, $394^{* *}$ &, $140^{*}$ \\
POS_SAT3 & & &, $162^{*}$ \\
\hline
\end{tabular}

Nota. $^{*}$ Não nulas e significativas a p<0,05. ${ }^{* *}$ Não nulas e significativas a $\mathrm{p}<0,01$. Fonte: Dados da pesquisa (2019).

A consistência interna, por sua vez, apresentou o resultado de 0,515 e a variância explicada em 43,52\%, inicialmente. Como forma de melhorar os resultados, o item POS_SAT4 foi deletado, pois apresentava escore $(0,302)$ e comunalidade $(0,146)$ baixos.

Tabela 18: Medidas da análise fatorial do estágio Pós-compra, dimensão Satisfação

\begin{tabular}{clcc}
\hline \multirow{2}{*}{ CóD } & & \multicolumn{2}{c}{$\begin{array}{c}\text { Variância: 55,78\% } \\
\text { Alpha: 0,603 }\end{array}$} \\
\cline { 3 - 4 } & & Item & Comu \\
\hline POS_SAT1 & Eu costumo ficar satisfeito com o produto comprado & 0,493 \\
POS_SAT2 & Eu costumo ficar satisfeito com o atendimento & 0,643 & 0,702 \\
POS_SAT3 & Eu costumo ficar satisfeito com o ambiente no qual compro artesanato & 0,802 \\
POS_SAT4 & Eu costumo ficar satisfeito com as opções de pagamento & 0,537 & 0,733 \\
\hline
\end{tabular}

Nota. *Item deletado. Fonte: Dados da pesquisa (2019). 
Com a exclusão, o teste de adequação da amostra $(\mathrm{KMO}=0,618)$ de esfericidade de Bartlett $\left(\chi^{2}=\right.$ $66,592 \mathrm{gl}=3$, a $\mathrm{p}<0,000$ ) sinalizaram que os itens estavam adequados para a análise fatorial. A variância alcançou o nível de 55,78\% e o alpha de Cronbach chegou a 0,603. Os resultados completos estão dispostos na Tabela 18.

\section{Dimensão Promoção}

Para a dimensão Promoção, mensurada inicialmente por seis itens, as correlações mais problemáticas ficaram em torno da variável POS_DIV5, que foram todas nulas e muito baixas. As demais foram significativas e ficaram em níveis moderados (acima de 0,400), com exceção da correlação POS_DIV1 x POS_DIV6 que obteve valor de 0,398 . Os dados estão na Tabela 19, a seguir.

Tabela 19: Matriz de correlação do estágio Pós-compra, dimensão Divulgação

\begin{tabular}{lccccc}
\hline & POS_DIV2 & POS_DIV3 & POS_DIV4 & POS_DIV5 & POS_DIV6 \\
\hline POS_DIV1 &, $598^{* *}$ &, $483^{* *}$ &, $515^{* *}$ &,- 103 &, $398^{* *}$ \\
POS_DIV2 & &, $648^{* *}$ &, $501^{* *}$ &, 039 &, $466^{* *}$ \\
POS_DIV3 & &, $646^{* *}$ &, 111 &, $526^{* *}$ \\
POS_DIV4 & & &, 080 &, $432^{* *}$ \\
POS_DIV5 & & & & &,- 018 \\
\hline
\end{tabular}

Nota. $^{* *}$ Não nulas e significativas a p<0,01. Fonte: Dados da pesquisa (2019).

A análise de consistência interna indicou um valor de 0,753 , entretanto com a indicação de aumento para 0,842 caso a variável POS_DIV5 fosse excluída. Nesse sentido, procedemos à sua exclusão. 0 teste de adequação da amostra (KMO) ficou em 0,809 e de esfericidade de Bartlett com $\chi^{2}=418,041$ $\mathrm{gl}=10$, a $\mathrm{p}<0,000$. A variância extraída, por sua vez, alcançou o valor de $61,92 \%$. Os dados completos estão dispostos na Tabela 20.

Tabela 20: Medidas da análise fatorial do estágio Pós-compra, dimensão Divulgação

\begin{tabular}{|c|c|c|c|}
\hline \multirow[t]{2}{*}{ CóD } & \multirow[t]{2}{*}{ Item } & \multicolumn{2}{|c|}{$\begin{array}{c}\text { Variância: } 61,92 \% \\
\text { Alpha: } 0,753 \\
\end{array}$} \\
\hline & & Comu & Esc \\
\hline POS_DIV1 & Eu costumo recomendar produtos artesanais para familiares & 0,576 & 0,759 \\
\hline POS_DIV2 & Eu costumo recomendar produtos artesanais para amigos & 0,679 & 0,824 \\
\hline POS_DIV3 & Eu costumo indicar o artesão para amigos & 0,722 & 0,850 \\
\hline POS_DIV4 & Eu costumo indicar o artesão para familiares & 0,624 & 0,790 \\
\hline POS_DIV5 & Faço propaganda negativa dos produtos artesanais quando não gosto & $*$ & $*$ \\
\hline POS_DIV6 & Eu costumo divulgar o trabalho do artesão nas redes sociais. & 0,494 & 0,703 \\
\hline
\end{tabular}

Nota. *Item deletado. Fonte: Dados da pesquisa (2019).

\section{Passo 7 - Atividades de campo adicionais}

Seguindo os passos para validação da escala, após os ajustes do passo anterior, efetuamos uma segunda coleta de dados com o intuito de proceder à Análise Confirmatória da Escala (AFC). A nova coleta ocorreu entre os meses de maio e junho de 2019, obtendo 520 respostas, sendo 144 coletadas presencialmente e 376 online. A coleta presencial seguiu os mesmos parâmetros indicados nos primeiros procedimentos de limpeza da escala. O questionário também foi estruturado na plataforma Survey Monkey e o link foi compartilhado em redes sociais, Facebook, Instagram e Whatsapp. A pergunta filtro consistia em saber se o respondente consumiu ou consome objetos artesanais.

\section{Passo 8 - Procedimento de limpeza de escalas adicionais}

Como primeira etapa foram realizados procedimentos de limpeza adicional, inicialmente efetuamos uma análise exploratória. Dos 520 questionários iniciais, 78 foram descartados, pois não estavam completamente respondidos. Assim, a amostra total da segunda coleta é composta por 442 respondentes, destes $61,3 \%$ são mulheres, $36,4 \%$ homens e $2,3 \%$ da amostra se divide entre transgênero e nãobinário. A idade média dos respondentes foi 31,47 anos, com desvio padrão de 11,67. Quanto à escolaridade, os maiores percentuais se concentram entre ensino médio $(25,1 \%)$, superior $(28,1)$ e pós-graduação $(33,9 \%)$ completos. Diferente da primeira amostra, observou-se maior equilíbrio entre as faixas de renda mensal, na faixa até um salário mínimo compreende $28,5 \%$ dos respondentes e $21,9 \%$ possuem renda variando entre maior que três a dez salários mínimos. Na segunda amostra, também se observa maior variedade em relação à cidade e estado onde os respondentes residem. Enquanto a primeira coleta ficou limitada aos estados do Ceará e Pernambuco, na segunda coleta observa-se respondentes do 
Rio Grande do Norte (19), Minas Gerais (9), Bahia (14), Maranhão (4), Paraíba (13), São Paulo (10), entre outros que apresentaram menor quantidade de respondentes.

Após a finalização da análise exploratória dos dados, realizamos a Análise Fatorial Confirmatória. Como boa parte das dimensões ficaram com três itens, a AFC ficou comprometida. Devido às limitações de espaço, apresentaremos os principais resultados e as justificativas para decisões tomadas com o intuito de obter as melhores métricas. Para a AFC, foi utilizado o software AMOS (versão 22). Os parâmetros para a referida análise seguiram as recomendações propostas em Costa (2011), sendo: divisão do Qui-Quadrado $\left(\chi^{2}\right)$ pelo número de Graus de liberdade (gl) $<=5$; GFI $>=0,9$; CFI $>=0,9$ e RMSEA $<=0,08$.

\section{Estágio Pré-compra}

Para esse estágio, as dimensões de Identidade, Finalidade e Artesão não atenderam aos requisitos mínimos dos parâmetros. De modo que consideramos as dimensões inválidas. Houve a tentativa de verificar, por meio de uma análise fatorial, se pelo menos quatro itens se agrupariam em um único fator, no intuito que uma possível nova dimensão surgisse. Entretanto, isso não ocorreu, de modo que decidimos encerrar as análises para as três dimensões.

\section{Dimensão Influência dos grupos de referência}

A dimensão relativa à Influência dos grupos de referência ficou com os quatro itens provenientes da primeira etapa de limpeza. 0 modelo alcançou boas medidas em todos os parâmetros de análise já na primeira interação, de modo que não efetuamos novos ajustes. Os dados completos para a dimensão estão dispostos na Tabela 21.

Tabela 21: Resultados da Análise Fatorial Confirmatória estágio Pré-compra, dimensão Influência dos grupos de Referência

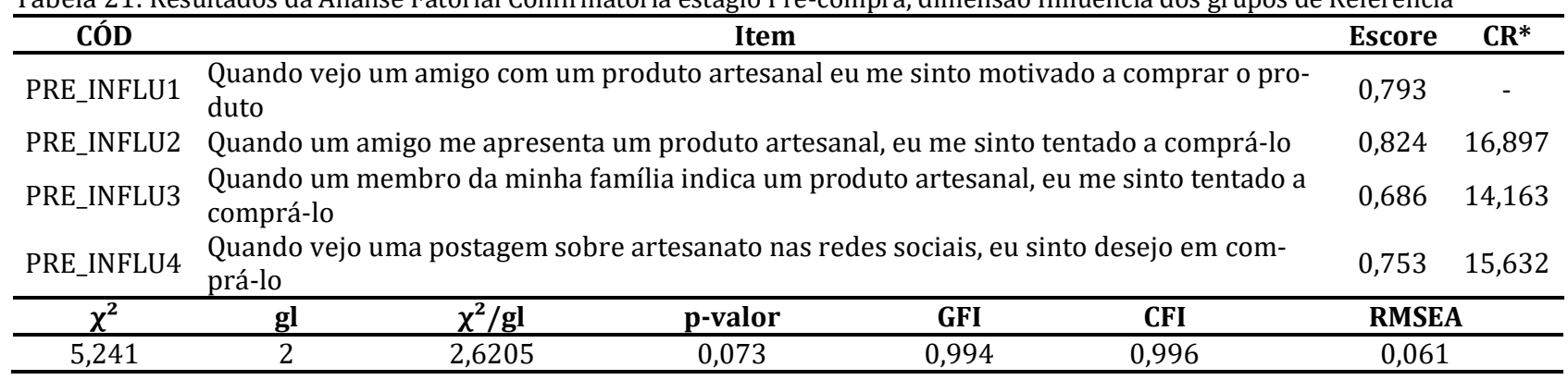

Nota. *Todos significativos à p-valor<0,001. Fonte: Dados da Pesquisa (2019).

\section{Dimensão Características do produto}

Para essa dimensão, foi necessário fazer três interações até ajustarmos o modelo dentro dos parâmetros necessários. 0 modelo inicial com as seis variáveis provenientes da primeira etapa apresentou duas variáveis problemáticas (PRE_CAR6 e PRE_CAR9), de modo que procedemos às exclusões dos itens. Os parâmetros, apesar de apresentarem valores bons em quase todas as medidas, obtiveram melhores resultados após a retirada desses dois itens. Na Tabela 22 pode-se verificar os dados.

Tabela 22: Resultados da Análise Fatorial Confirmatória estágio Pré-compra, dimensão Características do bem material

\begin{tabular}{|c|c|c|c|c|c|c|}
\hline CóD & \multicolumn{4}{|c|}{ Item } & Escore & $\mathbf{C R}^{*}$ \\
\hline PRE_CAR3 & \multicolumn{4}{|c|}{ Eu compro artesanato por não ser uma produção industrial massificada } & 0,615 & - \\
\hline PRE_CAR4 & \multicolumn{4}{|c|}{ Eu compro artesanato por envolver uma habilidade técnica repassada por gerações } & 0,699 & 9,561 \\
\hline PRE_CAR5 & \multicolumn{4}{|c|}{ Eu compro artesanato por envolver habilidade manual } & 0,650 & 9,338 \\
\hline PRE_CAR7 & \multicolumn{4}{|c|}{ Eu compro artesanato porque é sustentável } & 0,579 & 8,748 \\
\hline$\chi^{2}$ & $\chi^{2} / g l$ & p-valor & GFI & CFI & \multicolumn{2}{|c|}{ RMSEA } \\
\hline 7,498 & 3,749 & 0,024 & 0,994 & 0,984 & \multicolumn{2}{|c|}{0,079} \\
\hline
\end{tabular}

Nota. ${ }^{*}$ Todos significativos à p-valor $<0,001$. Fonte: Dados da Pesquisa (2019).

\section{Estágio Momento da compra}

As dimensões do estágio no momento da compra também apresentaram problemas na Análise Fatorial Confirmatória, de modo que excluímos as dimensões Preço e Produto. A única dimensão com resultados dentro dos parâmetros foi a de Serviço, mensurada por quatro itens. A única ressalva foi o escore da variável COM_SER5 que ficou em 0,277. Apesar de ser um valor baixo, decidimos manter o item considerando que o Critical Ratio foi de 4,191 e significativo. Na Tabela 23, se pode verificar os 
dados.

Tabela 23: Resultados da Análise Fatorial Confirmatória estágio Momento da compra, dimensão Serviço

\begin{tabular}{|c|c|c|c|c|c|c|}
\hline CóD & \multicolumn{4}{|c|}{ Item } & Escore & CR* \\
\hline COM_SER1 & \multicolumn{4}{|c|}{ A forma como o objeto é exposto me incentiva a comprá-lo } & ,549 & - \\
\hline COM_SER2 & \multicolumn{4}{|c|}{ Eu observo o atendimento prestado no momento da compra do artesanato } & 618 & 6,571 \\
\hline COM_SER3 & \multicolumn{4}{|c|}{ Costumo levar mais produtos artesanais quando sou bem atendido } &, 593 & 6,608 \\
\hline COM_SER5 & \multicolumn{4}{|c|}{ Eu observo o conforto do ambiente de compra para a aquisição do objeto artesanal } & 277 & 4,191 \\
\hline$\chi^{2}$ & $\chi^{2} / \mathrm{gl}$ & p-valor & GFI & CFI & \multicolumn{2}{|c|}{ RMSEA } \\
\hline 5,460 & 2,730 & 0,065 & 0,994 & 0,979 & \multicolumn{2}{|c|}{0,063} \\
\hline
\end{tabular}

Nota. ${ }^{*}$ Todos significativos à p-valor<0,001. Fonte: Dados da Pesquisa (2019).

\section{Estágio Pós compra}

No último estágio, a dimensão Satisfação também apresentou problemas em seu modelo, de modo que também optamos por excluí-la. A outra dimensão versava sobre a Divulgação. 0 modelo formado por cinco itens resultou em problemas em três medidas $\chi^{2} / g \mathrm{l}=21,482$; $\mathrm{CFI}=0,859$ e RMSEA=0,216. Com a exclusão do item POS_DIV1, o modelo ainda apresentou algumas medidas fora dos parâmetros aceitáveis. No entanto, tomamos o modelo como aceitável, conforme dados da Tabela 24.

Tabela 24: Resultados da Análise Fatorial Confirmatória estágio Pós-compra, dimensão Divulgação

\begin{tabular}{|c|c|c|c|c|c|c|c|}
\hline CóD & \multicolumn{5}{|c|}{ Item } & Escore & $\mathbf{C R}^{*}$ \\
\hline POS_DIV2 & \multicolumn{5}{|c|}{ Eu costumo recomendar produtos artesanais para amigos. } & 0,611 & - \\
\hline POS_DIV3 & \multicolumn{5}{|c|}{ Eu costumo indicar o artesão para amigos. } & 0,845 & 12,995 \\
\hline POS_DIV4 & \multicolumn{5}{|c|}{ Eu costumo indicar o artesão para familiares. } & 0,854 & 13,031 \\
\hline POS_DIV6 & \multicolumn{5}{|c|}{ Eu costumo divulgar o trabalho do artesão nas redes sociais. } & 0,652 & 11,047 \\
\hline$\chi^{2}$ & gl & $\chi^{2} / \mathrm{gl}$ & p-valor & GFI & CFI & \multicolumn{2}{|c|}{ RMSEA } \\
\hline 12,937 & 2 & 6,469 & 0,02 & 0,986 & 0,984 & \multicolumn{2}{|c|}{0,111} \\
\hline
\end{tabular}

Nota. ${ }^{*}$ Todos significativos à p-valor<0,001. Fonte: Dados da Pesquisa (2019).

\section{Passo 9 - Análise de validade e de confiabilidade da escala final}

Neste passo, verificamos a validade de construto a partir da validade convergente e discriminante. Para o primeiro caso, alcançamos a validade convergente, pois todos os valores dos Critical Rations são significativos ao nível 0,001 . Já para o caso da validade discriminante, procedemos ao cálculo da variância extraída e compartilhada, sendo esta última medida pelo quadrado da correlação de Pearson. Segundo Costa (2011), se a variância compartilhada for menor que a variância extraída temos evidência de validade discriminante. Dessa forma, ao analisar a Tabela 25, verificamos que a variância extraída (representada na linha diagonal em negrito) é superior à variância compartilhada, mostrando validade discriminante.

Ainda de acordo com os dados da Tabela 25, ao verificar o valor da consistência interna das dimensões, notamos que com exceção da dimensão Serviço, todas as demais apresentaram valores bons, ficando acima de 0,700. Já quando analisamos a confiabilidade composta, mais uma vez a dimensão Serviço apresentou problemas, pois o valor mínimo para esse tipo de confiabilidade é de 0,700 (Costa, 2011).

Tabela 25: Resultados de Confiabilidade e Validade

\begin{tabular}{lccccc}
\hline \multicolumn{1}{c}{ Dimensão } & Alpha & $\begin{array}{c}\text { Confiabilidade } \\
\text { Composta }\end{array}$ & \multicolumn{3}{c}{ Variância extraída e Compartilhada } \\
\hline Grupos de Referência & 0,848 & 0,849 & $\mathbf{0 , 6 5 8}$ & & \\
Características do Produto & 0,728 & 0,731 & 0,332 & $\mathbf{0 , 5 5 3}$ & $\mathbf{0 , 4 4 7}$ \\
Serviço & 0,540 & 0,590 & 0,082 & 0,122 & 0,066 \\
Divulgação & 0,821 & 0,833 & 0,398 & 0,361 & 0,685 \\
\hline
\end{tabular}

Fonte: Dados da pesquisa (2019).

\section{Passo 10 - Desenvolvimento de normas e recomendações de uso e interpretação}

No último passo, são elencadas as instruções para aplicação da escala. Recomendamos que sejam seguidas as instruções já consolidadas na literatura (cf Costa, 2011), como dispor aleatoriamente os itens, evitando deixar no mesmo bloco variáveis de uma mesma dimensão.

A escolha do tipo de escala de verificação (ex: Likert ou Phrase Completion) e do número de pontos (ex: $5,7,10$ ou 11) compete a cada pesquisador que utilizar a escala, que o fará seguindo suas orientações teóricas e metodológicas em pesquisas quantitativas. A interpretação de medidas de posição e dispersão devem ser feitas considerando o número de pontos da escala. 
Por fim, a escala pode ser usada para mensuração do consumo de objetos artesanais de maneira geral, mas também em contexto mais específico, a exemplo de tipologias (ex: madeira), desde que as devidas adaptações sejam feitas. Em relação à dimensão Serviço, recomendamos que seja dada uma atenção especial devido aos problemas da confiabilidade e validade.

\section{Considerações finais}

Este estudo teve como objetivo desenvolver e validar uma escala para consumo de objetos artesanais. Para isso, seguimos os dez passos propostos por Costa (2011) para o desenvolvimento de escalas. A escala final é composta por quatro dimensões, cada uma com quatro itens e é apresentada na Figura 4.

\begin{tabular}{|c|c|l|}
\hline Estágio & Dimensão & \multicolumn{1}{c|}{ Item } \\
\hline \multirow{2}{*}{$\begin{array}{c}\text { Pré-com- } \\
\text { pra }\end{array}$} & $\begin{array}{c}\text { Influência do } \\
\text { grupo de Refe- } \\
\text { rências }\end{array}$ & $\begin{array}{l}\text { Quando vejo um amigo com um produto artesanal eu me sinto motivado a comprar o produto } \\
\text { Quando um amigo me apresenta um produto artesanal, eu me sinto tentado a comprá-lo } \\
\text { Quando um membro da minha família indica um produto artesanal, eu me sinto tentado a } \\
\text { comprá-lo } \\
\text { Quando vejo uma postagem sobre artesanato nas redes sociais, eu sinto desejo em comprá- } \\
\text { lo }\end{array}$ \\
\cline { 2 - 4 } & $\begin{array}{c}\text { Características } \\
\text { do bem mate- } \\
\text { rial }\end{array}$ & $\begin{array}{l}\text { Eu compro artesanato por não ser uma produção industrial massificada } \\
\text { Eu compro artesanato por envolver uma habilidade técnica repassada por gerações } \\
\text { Eu compro artesanato por envolver habilidade manual } \\
\text { Eu compro artesanato porque é sustentável }\end{array}$ \\
\hline $\begin{array}{c}\text { Momento } \\
\text { da com- } \\
\text { pra }\end{array}$ & Serviço & $\begin{array}{l}\text { A forma como o objeto é exposto me incentiva a comprá-lo } \\
\text { Eu observo o atendimento prestado no momento da compra do artesanato } \\
\text { Costumo levar mais produtos artesanais quando sou bem atendido } \\
\text { Eu observo o conforto do ambiente de compra para a aquisição do objeto artesanal }\end{array}$ \\
\hline $\begin{array}{c}\text { Pós-com- } \\
\text { pra }\end{array}$ & Divulgação & $\begin{array}{l}\text { Eu costumo recomendar produtos artesanais para amigos. } \\
\text { Eu costumo indicar o artesão para amigos. } \\
\text { Eu costumo indicar o artesão para familiares. } \\
\text { Eu costumo divulgar o trabalho do artesão nas redes sociais. }\end{array}$ \\
\hline
\end{tabular}

Figura 4: Escala final para Consumo de objetos artesanais

Fonte: elaborado pelos autores (2019).

Em relação às implicações gerenciais, esse instrumento contribui especialmente para o conhecimento sobre o consumo de um produto cultural tão característico e específico, bem como se constitui uma alternativa para os profissionais que queiram saber mais sobre o consumo de suas peças e para gestores públicos que queiram realizar pesquisas relacionadas ao turismo. Como limitações desse estudo, citamos a origem de respondentes, restritos basicamente à região Nordeste e com escolaridade em nível de pós-graduação, o que pode ter contribuído para resultados não satisfatórios para boa parte das dimensões inicialmente propostas. Assim, recomenda-se que novas pesquisas sejam direcionadas para o conhecimento do consumo em outras regiões do país. Não obstante, incentiva-se também a inclusão de novas dimensões para uma melhor compreensão do consumo de objetos artesanais.

\section{Referências}

Artoni, F. L.; Daré, P. R. C. (2008)Reputação corporativa e a comunicação boca-a-boca: uma interdependência inequívoca. Revista Pretexto, 9(1), 33-50.

Barbosa, L.; Campbell, C. (2006). 0 estudo do consumo nas ciências sociais contemporâneas. Cultura, consumo e identidade. Rio de Janeiro: Editora FGV, 21-44.

Belk, R. W. (1988). Possessions and the extended self. Journal of consumer research, 15(2), 139-168.

Bento, K. D.; Silva, I. F. O. (2016). Selo de autenticidade do artesanato do Estado do Ceará: a fusão entre uma ferramenta de marketing e uma estratégia de desenvolvimento local. Revista Brasileira de Gestão e Desenvolvimento Regional, 12(4), 260-283.

Bragaglia, A. P. (2010). Comportamentos de consumo na contemporaneidade. Comunicação Mídia e Consumo, 7(19), 107-124.

Campbell, C. (2006). Eu compro, logo sei que existo: as bases metafísicas do consumo moderno. Cultura, consumo e identidade. Rio de Janeiro: FGV, 47-64.

Canclini, N. G. (1983). As culturas populares no capitalismo. São Paulo: Brasiliense. 
Chagas Neto, B. (2006). Consumo para obtenção de status: estudo empírico entre meninas pré-adolescentes de São Luís do Maranhão. 2006. (Dissertação de Mestrado em Administração. EBAPE. São Luiz: EBAPE).

Cleps, G. D. G. (2004). 0 comércio e a cidade: novas territorialidades urbanas. Revista Sociedade \& Natureza, v. 16 (30), 117-132.

Costa, F. J. (2011). Mensuração e desenvolvimento de escalas: aplicações em administração. Rio de Janeiro: Ciência Moderna.

Giese, J. L.; Cote, J. A. (2000). Defining consumer satisfaction. Academy of marketing science review, $1(1), 1-22$.

Girón, J. P. H.; Hernández, M. L. D.; Castañeda, J. C. J. (2007). Strategy and factors for success: The mexican handicraft sector. Performance Improvement, 46(8), 16-26.

IBGE. (2015). Perfil dos estados e dos municípios brasileiros: cultura 2014, Coordenação de População e Indicadores Sociais Rio de Janeiro: IBGE.

Katz-Gerro, T. (2002). Highbrow cultural consumption and class distinction in Italy, Israel, West Germany, Sweden, and the United States. Social forces, 81(1), 207-229.

Keller, P. F. (2014). 0 artesão e a economia do artesanato na sociedade contemporânea. Politica \& Trabalho, 41, 323-347.

Lages, V.; Lagares, L.; BRAGA, C. L. (Org.). (2006). Valorização de produtos com diferencial de qualidade e identidade: indicações geográficas e certificações para a competitividade nos negócios. Brasília: SEBRAE.

Lima, R. G. (2009). Artesanato e arte popular: duas faces de uma mesma moeda. Brasília: Ministério da Cultura - Centro Nacional de Folclore e Cultura Popular.

Marchetti, R.; Prado, P. H. M. (2001). Um tour pelas medidas de satisfação do consumidor. Revista de administração de empresas, 41(4), 56-67.

Meitiana, M.; Setiawan, M.; Rohman, F.; Irawanto, D. (2019). Factors affecting souvenir purchase behavior: valuable insight for tourism marketers and industry. Journal of Business and Retail Management Research, 13(3), 248-255.

Mendoza-Ramírez, L.; Toledo-López, A. (2014). Strategic orientation in handicraft subsistence businesses in Oaxaca, Mexico. Journal of Marketing Management, 30(5-6), 476-500.

Montoro-Pons, J. D.; Cuadrado-García, M. (2018). Religiosity and cultural consumption. International journal of consumer studies, 42(6), 704-714.

Novaes, A. M. C. (2011). O processo de artificação em Juazeiro do Norte. Análise do Centro Cultural Mestre Noza. Tese de doutorado. Universidade Federal do Ceará.

Pani, D.; Pradhan, S. K. (2016). An Empirical Study of Impact of Demographic Variables on Consumer Preference towards Tribal Handicraft-A Case of Rayagada District during Chaiti Festival. Pacific Business Review International, 8(7), 61-68.

Rabêlo Neto, A. et al. (2014). Os antecedentes do consumo de produtos culturais por consumidores de baixa renda. Revista Brasileira de Marketing, 13(1), 75-92.

Reeves, A.; DE Vries, R. (2019). Can cultural consumption increase future earnings? Exploring the economic returns to cultural capital. The British journal of sociology, 70(1), 214-240.

Scrase, T. J. (2003). Precarious production: globalisation and artisan labour in the Third World. Third World Quartely, 24(3), 449-461.

Sharda, N.; Bhat, A. K. (2018). Austerity to materialism and brand consciousness: luxury consumption in India. Journal of Fashion Marketing and Management: An International Journal, 22(2), 223-239, 2018.

Silva, F. B. da; Araújo, H. E.; Souza, A. L. (2007). 0 consumo cultural das famílias brasileiras. In: Silveira, F. G. et al (Orgs.). Gasto e consumo das famílias brasileiras contemporâneas, Brasília: IPEA, 2, 105-142.

Silver; G.; Kundu, P. K. (2013). Handicraft products: Identify the factors that affecting the buying decision of customers (The viewpoints of Swedish shoppers). Dissertação de Mestrado, Umea University.

Sirdeshmukh, D.; Singh, J.; Sabol, B. (2002). Consumer trust, value, and loyalty in relational exchanges. Journal of marketing, 66(1), 15-37.

Solomon, M. R. (2016). O comportamento do consumidor : comprando, possuindo e sendo. Porto Alegre: Bookman. 